\section{Exacerbation of generalized plaque psoriasis after tuberculin test}

\author{
Alberto Corrà,, ${ }^{1}$ Lavinia Quintarelli, ${ }^{1}$ \\ Alice Verdelli, ${ }^{2}$ Volpi Walter, ${ }^{1}$ \\ Marzia Caproni ${ }^{1}$
}

${ }^{1}$ Section of Dermatology, Department of Health Sciences, University of Florence; ${ }^{2}$ Department of Experimental and Clinical Biomedical Sciences "Mario Serio", University of Florence, Italy

\begin{abstract}
Koebner phenomenon, also known as isomorphic response, is the appearance of active skin lesions in sites of epidermal injury. This manifestation is characteristic of different dermatological diseases, such as lichen planus, vitiligo and psoriasis. We present the case of a psoriatic patient who experienced Koebner phenomenon after Mantoux test, followed by eruption of generalized plaque psoriasis.
\end{abstract}

\section{Introduction}

Biologic drugs have revolutionized the treatment of moderate to severe psoriasis, with a well-known increased risk of tuberculosis (TB) reactivation. In order to avoid this risk, screening for latent tuberculosis infection (LTBI) is essential to prevent the risk of active TB. Despite several disadvantages, tuberculin skin test (TST) still represents one of the most useful tools for LTBI detection. In this paper, we report the case of a 61-year-old female who experienced a severe relapse of psoriasis after Mantoux test.

\section{Case report}

A 61-year-old female presented with a 7-year history of severe arthropathic plaque psoriasis. The patient was also affected by mild hypercholesterolaemia. In the last years, she has been treated with different therapies including UVB-narrowband phototherapy, methotrexate, cyclosporine and etanercept. Cyclosporine revealed to be the best effective approach for the patient, but it was discontinued for arterial hypertension onset, while etanercept has revealed ineffective in a 3-months treatment course. Her past medical history included also an episode pneumococcal pneumonia occurred several years before, successfully treated with antibiotic therapy. At the time of visit, the patient showed several well-defined plaques, characterized by mild infiltration, moderate erythema and scaling, involving trunk and principally extensor surfaces of limbs, with a Psoriasis Area Severity Index (PASI) score of 18.2. Since the patient had previously shown to be unresponsive to TNF- $\alpha$-inhibitors, we considered ustekinumab in accordance with rheumatologists. Before starting treatment, we collected personal history, physical examination, and laboratory tests. On infectious diseases specialist's indication, screening for HIV, TB and viral hepatitis were also performed. A chest radiograph showed calcifications and scarring consistent with previous infection, while Mantoux test (MT) performed on her left forearm revealed a negative reaction. Other investigations were all within normal limit, including Quantiferon $\square \mathrm{TB}$ Gold test. After four days, the patient developed a psoriatic plaque at the site of injection, while a severe worsening of psoriasis was observed, with comparison of new lesions and enlargement of the pre-existing ones with a PASI score of 28 (Figure 1).

The lesions were deeply infiltrated and mildly scaly. A skin biopsy showed confluent areas of epidermal hyperplasia with parakeratosis, moderate inflammatory infiltrate in the upper dermis with scattered eosinophils and dilated small vessels in the papillary dermis. Histopathological data confirmed the clinical diagnosis of generalized plaque psoriasis. Patient started topical treatment with calcipotriol and betamethasone ointment, in addition to systemic ustekinumab (45 mg every three months) which revealed effective in reducing psoriatic lesions with PASI score of 3.2 at the successive 3 months follow-up.

\section{Discussion and conclusions}

The MT is a screening tool for LTBI, consisting in subdermal injection of purified proteins derivative (PPD), obtained from stains of $M$. tubercolosis. This screening test is commonly performed to exclude LTBI in patients eligible for immunosuppressive treatment, while it is not reliable for patients already in treatment with antiTNF drugs, ${ }^{1,2}$ since TNF is a key-cytokine in tuberculin-hypersensitivity reaction development and its inhibition can lead to false-negative results. IGRA (interferonrelease assay) is a test measuring specific Tcell-based responses to M. tubercolosisspecific antigens including ESAT-6, CFP10 and can be used in addition to MT or in alternative to it. ${ }^{3}$ Since MT produces a epi-
Correspondence: Alberto Corrà, Section of Dermatology, Department of Health Sciences, University of Florence, Viale Michelangiolo 41, 50125 Florence, Italy.

E-mail: alberto.corra@unifi.it

Key words: Koebner phenomenon, Latent Tubercolosis Infection, Plaque psoriasis, Mantoux test.

Contributions: AC: contributed collecting clinical data, writing the manuscript and finally approving the work; LQ contributed collecting clinical data, revising critically and approving the final version; AV: contributed in design and interpretation of the work, revising and approving the final version; WV contributed in data analysis, revising and approving the final version; MC: contributed in design, interpretation and analysis of the work, revising it critically and approving the final version.

Conflict of interest: The authors declare no potential conflict of interest.

Funding: None.

Ethical approval and informed consent: Informed consent was obtained.

Availability of data and material: Data and materials are available by the authors.

Please cite this article as: Corrà A, Quintarelli L, Verdelli A, et al. Exacerbation of generalized plaque psoriasis after tuberculin test. Dermatol Rep 2021;13:9019.

Received for publication: 14 November 2020. Revision received: 6 March 2021.

Accepted for publication: 17 March 2021.

This work is licensed under a Creative Commons Attribution-NonCommercial 4.0 International License (CC BY-NC 4.0).

${ }^{\circ}$ Copyright: the Author(s), 2021

Licensee PAGEPress, Italy

Dermatology Reports 2021; 13:9019

doi:10.4081/dr.2021.9019

dermal rupture, a Koebner phenomenon, or isomorphic response, can be induced. Indeed, psoriatic patients develop active skin lesions at sites of skin injury, even if previously unaffected. Skin injuries may be represented by trauma, infective or inflammatory diseases, sunburn, or medicaments. ${ }^{4}$ In literature, we found few other cases of Koebner phenomenon in which a psoriatic plaque developed after MT in the site of injection of.$^{5-7}$ One of the patients reported was previously unaffected by psoriasis. In these cases, the lesions appeared within 3 days from the injection, a latency similar to 


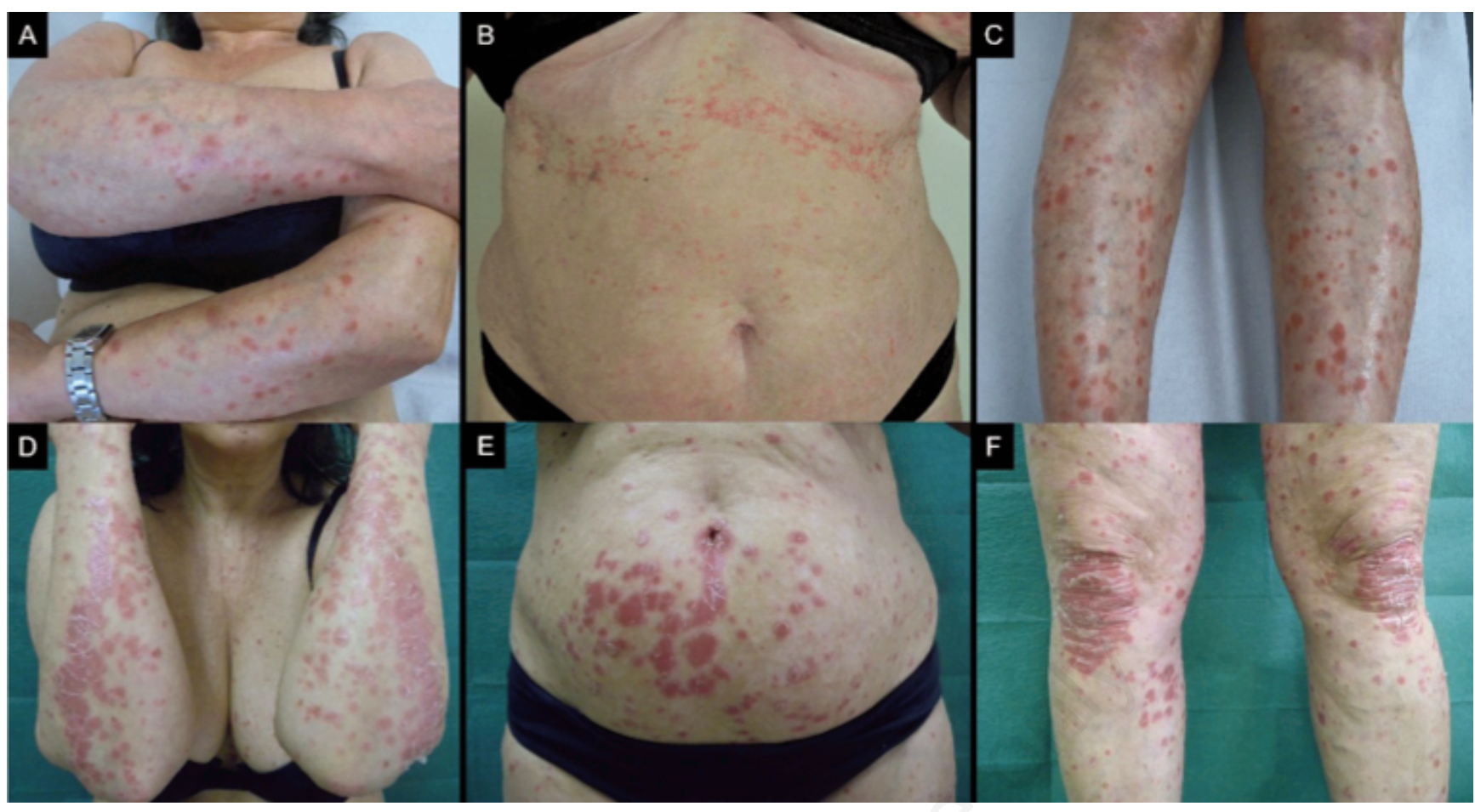

Figure 1. A-C) small erythematous scaly lesions on arms, elbows, abdomen and legs before the Mantoux test. D-F) Pictures taken one week after the tuberculin test show enlargement of pre-existent lesions and appearance of new larger erythematous, scaly plaques in sites not previously involved.

the one of the patients described in our case. However, this phenomenon may happen more frequently than reported in literature, according to a study in which PPD seems to be more effective in inducing koebnerization than injury alone. ${ }^{8}$ On the contrary, other works showed opposite findings, ${ }^{9}$ so this aspect is not fully understood. Concerning the physiopathology, some Authors suggested that the upregulation of pro-inflammatory cytokines (such as interferon- $\alpha$ and TNF- $\alpha$ ) following epidermal injury could lead to inflammatory infiltration in the site of tuberculin injection and activate pathogenic processes involved in the development of psoriatic plaque in predisposed patients. The Koebner phenomenon would not explain the appearance of generalized lesions few days after the tuberculin test. The causal relationship between PPD injection and exacerbation of psoriasis was suspected on the basis of the compatible timing, while a spontaneous worsening of the disease could not be excluded considering the unpredictability of psoriasis course. Similar reports are scarce in literature: a case of generalized pustular psoriasis occurred after MT with a latency of 72 hours. $^{10}$ The timing is comparable to the relapse experienced by our patient, but this is still not sufficient to define a clear causality link. Tubercular antigens showed in vitro the capability to stimulate activation and proliferation of T-lymphocytes collected from inflammatory synovitis of patients with rheumatoid arthritis and psoriatic arthritis. Response to mycobacterial antigen may be secondary to cross-reactivity due to homology with human inflammatory proteins. ${ }^{11} \mathrm{~A}$ similar process may be responsible for the enhancement of inflammatory cutaneous response, leading to the worsening of disease in psoriatic patients. Conversely, peripheral mononuclear cells of psoriasis patients showed in vitro decreased responsiveness to mycobacterial antigens, compared to healthy controls. ${ }^{12}$ To date, the pathophysiology of this mechanism is still far from being fully comprehended. However, this seems to be an occurrence that, although rare, should be considered in the management of psoriasis.

\section{References}

1. Katsenos S, Nikolopoulou M, Tsiouri G et al. The challenging Evaluation of Patients with Severe Psoriasis for Latent Tuberculosis: An Important Indication for IGRA. Open Respir Med J 2011;5:59-60.

2. Haddican MM, Koo JY. Is tuberculin skin testing reliable during anti-tumor necrosis factor-alfa therapy? A case report and review of the literature. J Am Acad Dermatol 2011;65:195-7.

3. Auguste P, Tsertsvadze A, Pink J, et al. Comparing interferon-gamma release assays with tuberculin skin test for identifying latent tuberculosis infection that progresses to active tuberculosis: systematic review and meta-analysis. BMC Infect Dis 2017;17:200.

4. Wu JK, Siller G, Strutton G. Psoriasis induced by topical imiquimod. Australas J Dermatol 2004;45:47-50.

5. Sahin GO, Akyol M, Ozcelik S. An unusual Koebner phenomenon secondary to PPD with pustular and arthropathic psoriasis. Eur J Dermatol 2000;17:446.

6. Khanna D, Singal A, Gupta R. Onset of psoriasis triggered by Mantoux test. Indian J Dermatol Venereol Leprol 2014;80:451-4.

7. Piaserico S, Dan G, Peserico A. Koebner phenomenon in psoriasis induced by Mantoux test. J Eur Acad Dermatol Venereol 2009;23:1103-5.

8. Dogan B, Harmanyeri Y. Intradermal antigen tests and the Koebner phenomenon in psoriasis. Int $\mathrm{J}$ Dermatol 1997;36:263-5.

9. McFadden JP, Powles AV, Baker BS, et 
al. Intradermal antigen tests in psoriasis. 11. Pope RM, Wallis RS, Sailer D, et al. T Acta Derm Venereol. 1990; 70: 262-4.

10. Mendez J, Garcia I, Escudero G, et al. Generalized pustular psoriasis induced by tuberculin testing. Actas Dermosifilogr. 2007; 98: 507-8 cell activation by mycobacterial antigens in inflammatory synovitis. Cell Immunol 1991;133:95-108.

12. Bay ML, Lehrer A, Bressanelli A, et al. Psoriasis patients have T-cells with reduced responsiveness to common mycobacterial antigens. FEMS Immunol Med Microbiol 1998;21:6570 . 